\title{
The clinical significance of CCBEI expression in human colorectal cancer
}

This article was published in the following Dove Press journal:

Cancer Management and Research

\author{
Yan-Rong Zhao' \\ Hao Liu ${ }^{2}$ \\ Li-Miao $\mathrm{Xiao}^{3}$ \\ Can-Guang Jin' \\ Zhi-Peng Zhang' \\ Chun-Guang Yang ${ }^{4}$ \\ 'Department of General Surgery, \\ Xiangya Hospital, Central South \\ University, Changsha, Hunan, China; \\ ${ }^{2}$ Department of General Surgery, The \\ Second Xiangya Hospital, Central \\ South University, Changsha, Hunan, \\ China; ${ }^{3}$ Department of Ultrasound, \\ Hunan Children's Hospital, Changsha, \\ Hunan, China; ${ }^{4}$ Department of \\ Anesthesiology, Xiangya Hospital, \\ Central South University, Changsha, \\ Hunan, China
}

Purpose: The identification and discovery of prognostic markers for colorectal cancer (CRC) are of great clinical significance. CCBE1 is expressed in various tumors and its expression correlates with lymphangiogenesis and angiogenesis. However, the association between CCBE1 expression and $\mathrm{CRC}$ outcome has not been reported. The aim of this study was to investigate clinical significance of CCBE1 expression in CRC.

Patients and methods: CCBE1 expression was examined in 30 pairs of fresh CRC tissues and compared with adjacent normal (AN) tissues using quantitative real-time PCR (qRT-PCR), Western blotting and immunohistochemistry (IHC) staining. Tissue microarray immunohistochemical staining was used to study the CCBE1 expression characteristics of 204 CRC patient samples collected from January 2002 to December 2007, and the relationship of CCBE1 with clinicopathological features and prognosis of CRC was analyzed.

Results: CCBE1 was highly expressed in CRC tissues compared with matched AN tissues $(P=0.001)$. Moreover, high expression of CCBE1 was significantly associated with tumor differentiation, lymph node metastasis, vascular invasion, liver metastasis and TNM stage in CRC patients $(P \leq 0.01)$. Kaplan-Meier survival analysis revealed that high CCBE1 expression, poor tumor differentiation, lymph node metastasis and vascular invasion were significantly associated (all $P<0.001$ ) with poor prognosis for patients. Furthermore, univariate and multivariate Cox analysis revealed that high CCBE1 expression, poor tumor differentiation, lymph node metastasis and vascular invasion were independent risk factors for both overall survival (OS) and disease-free survival (DFS) of CRC patients (all $P<0.05$ ). OS and DFS of $267 \mathrm{CRC}$ patients from The Cancer Genome Atlas (TCGA) database showed the same trend (log-rank $P=6 \mathrm{e}-04$, HR [high] $=2.4$; log-rank $P=0.0081$, HR [high] $=1.9$ ).

Conclusion: High levels of CCBE1 contribute to the aggressiveness and poor prognosis of CRC. CCBE1 can serve as a novel potential biomarker to predict CRC patients' prognosis.

Keywords: CCBE1, prognosis, colorectal cancer, CRC, survival analysis, TCGA

\section{Introduction}

Colorectal cancer (CRC) is the third most common malignant cancer and one of the main causes of cancer-related death worldwide. There are approximately 1.4 million newly diagnosed CRC patients each year, and their 5-year survival rate is not ideal. ${ }^{1}$ The bad prognosis of patients with CRC is largely due to the metastatic progression. ${ }^{2}$ Metastasis is a multi-link, multifactorial, continuous and complex process that is one of the basic biological characteristics of malignant tumors and a key factor in determining the prognosis of cancer. ${ }^{3-5}$ In general, the metastatic process involves the spread
Correspondence: Chun-Guang Yang Department of Anesthesiology, Xiangya Hospital, Central South University, Xiangya Road 87, Changsha 410008, Hunan, China

Tel +86073I 84327413

Email chunguangy@yeah.net 
of tumor cells, vascular invasion, lymph node metastasis and growth of new cancer cell colonies. ${ }^{6,7} \mathrm{CRC}$ is one of the five leading causes of cancer-related death among both men and women in China. ${ }^{8}$ Currently, the incidence of CRC is still increasing in China, and approximately $25 \%$ of CRC patients have metastasized tumors prior to diagnosis. ${ }^{9}{ }^{910}$ Therefore, it is necessary to identify effective predictors associated with CRC progression and metastasis, which may help patients choose appropriate treatments and monitoring.

CCBE1, which is located in human chromosomal region $18 \mathrm{q} 21.32$, encodes a highly conserved protein with EGFlike domain. ${ }^{11}$ Early studies found that CCBE1 not only regulated extracellular matrix remodeling and migration and multicellular organism development ${ }^{12,13}$ but also played an important role in the development of lymphatic vessels, angiogenic sprouting and lymphangiogenic budding from venous endothelium. ${ }^{14-20}$ In the past few years, the role of CCBE1 in cancers is beginning to be reported. However, reports indicate that $\mathrm{CCBE} 1$ expression has different effects in different malignancies. Barton et $\mathrm{al}^{21}$ and $\mathrm{Li}$ et $\mathrm{al}^{22}$ supported CCBE1 as a potential tumor suppressor in ovarian cancer and lung cancer. Mesci et $\mathrm{al}^{23}$ and Mesci and $\mathrm{Liu}^{24}$ showed that in breast cancer CCBE1 is targeted by miR$330-3 p$, resulting in a more aggressive phenotype. However, Tian et $\mathrm{al}^{25}$ described CCBE1 as a tumor promoter in gastrointestinal stromal tumors (GISTs), which enhanced tumor angiogenesis. In addition, Guo et $\mathrm{al}^{26}$ and Zhang and $\mathrm{Liu}^{27}$ indicated that the increased expression of SLP-2 promoted the formation of lymph vessels and exacerbated lymphatic metastasis of rectal cancer via upregulation of CCBE1. Lymphatic metastasis and vascular invasion are the main means of CRC spread and can significantly affect the prognosis of patients. ${ }^{28-31}$ However, less information was available for the prognostic value of CCBE1 in CRC.

In this study, we detected the expression of CCBE1 in 30 cases of fresh CRC tissues and paired adjacent normal (AN) tissues and tissue samples from 204 CRC cases analyzed for correlation between CCBE1 expression and clinicopathological features. Further, whether CCBE1 could be used as a potential prognostic biomarker for primary CRC patients after surgical resection was assessed.

\section{Patients and methods}

\section{Patients and samples}

Thirty cases of fresh CRC tissues and matched AN tissues were collected from the Department of General Surgery from January to December 2017. All tissues were freshly frozen in liquid nitrogen until quantitative real-time PCR (qRT-PCR) and Western blotting test. Furthermore, from January 2002 to September 2007, a total of 204 patients' CRC samples with detailed clinical records were collected and reassembled into multiple tissue arrays in our retrospective study. All samples were collected without chemotherapy, immunotherapy and radiotherapy before surgery from Xiangya Hospital. All cases were diagnosed independently by two pathologists and followed up regularly until December 2017. This study was approved by the ethics committee of Xiangya Hospital of Central South University. According to the Declaration of Helsinki, all patients and their families provided written informed consent and agreed to use their tissue samples in the study. Follow-up of all the patients was carried out based on the surveillance suggested in the guidelines.

\section{RNA extraction and gene expression by qRT-PCR}

Total RNA from fresh CRC and AN tissues was extracted with the TRIzol reagent (Thermo Fisher Scientific, Waltham, MA, USA) according to the manufacturer's instructions. RNA quantity and quality were evaluated using a Nanodrop spectrophotometer (Thermo Fisher Scientific). RNA was reverse transcribed into cDNA by BeyoRT ${ }^{\mathrm{TM}}$ II First-Strand cDNA Synthesis Kit (Beyotime, Shanghai, China), and the expression of CCBE1 was measured using SYBR Green Master Mix (Beyotime) on the Applied Biosystems QuantStudio $^{\text {тм }} 3$ \& 5 Real-Time PCR System (Thermo Fisher Scientific). The sequences of the primers used were listed as follows: CCBE1-F: 5'-TACCGATATGACCGGGAGAG-3' and CCBE1-R: 5'-AGCTGCCCAAGGTATTGATG-3'; GADPH-F: 5'-GTCTCCTCTGACTTCAACAGCG-3' and GADPH-R: 5'-ACCACCCTGTTGCTGTAGCCAA-3'. The experiments were repeated three times. The data were normalized to GAPDH expression and calculated as $2^{-\Delta \Delta \mathrm{CT}}$.

\section{Western blot}

Proteins were extracted from fresh CRC and AN tissues using RIPA lysis buffer with protease inhibitors and quantified by BCA Protein Assay Kit (Beyotime). An amount of $30 \mu \mathrm{g}$ per sample was separated by SDS-PAGE and then transferred to the polyvinylidene difluoride (PVDF) membrane (Hoffman-La Roche Ltd., Basel, Switzerland). The membranes were blocked with 5\% skimmed milk and incubated with CCBE1 (Affinity, Cincinnati, OH, USA; diluted 1:1,000) and GAPDH (Affinity; diluted 1:1,000) antibodies overnight at $4{ }^{\circ} \mathrm{C}$. The antigen-antibody complex on the membrane was detected with enhanced chemiluminescence reagents (Thermo Fisher Scientific). 


\section{Immunohistochemistry (IHC)}

Tissue microarrays were constructed from a representative core from each CRC tissue and AN tissue. Tissue sections with diameters of $1.5 \mathrm{~mm}$ and $4 \mu \mathrm{m}$ thicknesses were placed on slides coated with 3-aminopropyltriethoxysilane. Immunohistochemical staining for tissue was performed using the polymer horseradish peroxidase detection system (Zhongshan Goldenbridge Biotechnology, Beijing, China). All tissue microarrays were incubated with the CCBE1 antibody (diluted 1:200) overnight at $4^{\circ} \mathrm{C}$. After incubation with secondary biotinylated antibody, sections were stained, the same length, with diaminobenzidine (DAB) and hematoxylin. The immunohistochemical staining intensity of CCBE1 was scored as negative (0), weak (1), moderate (2) and strong (3), and the percentage of positive cells was scored as 5\% (0), 5-30\% (1), 31-50\% (2) and $>50 \%$ (3). The scores were calculated by multiplying these two values (ranging from 0 to 9 ). The protein expression in CRC specimens was divided into the low expression group $(<4)$ and the high expression group $(\geq 4)$ for further analysis. ${ }^{32,33}$

\section{Statistical analyses}

All data were analyzed with IBM SPSS Statistics 22 Software (IBM Corporation, Armonk, NY, USA). Data were expressed as mean \pm standard error of the mean (SEM) for at least three independent experiments. Quantitative data between groups were compared using the Student's $t$-test. Correlations between different CCBE1 expression levels were determined using Pearson's chi-squared test. Overall survival (OS) and disease-free survival (DFS) analyses were performed by the Kaplan-Meier method and log-rank test. Univariate and multivariate analyses were analyzed with Cox proportional hazard regression model to verify the independent risk factors. $P$-value of $<0.05$ was defined as statistically significant.

\section{Results \\ Expression levels of CCBEI in CRC patients}

The expression of CCBE1 in 30 cases of fresh CRC tissues and matched AN tissues was detected by qRT-PCR, Western blotting and IHC. The qRT-PCR results indicated that CCBE1 mRNA level was significantly higher in CRC tissues compared with matched AN tissues ([fold change $\{\mathrm{CRC} /$ AN \} >2] in 63.3\% cases [19/30]; Figure 1A). Meanwhile, Western blotting results revealed that CCBE1 protein was highly expressed in most primary CRC tissues than in the matched AN tissues (Figure 1B). In addition, the immunohistochemical staining intensity of CCBE1 in liver metastatic
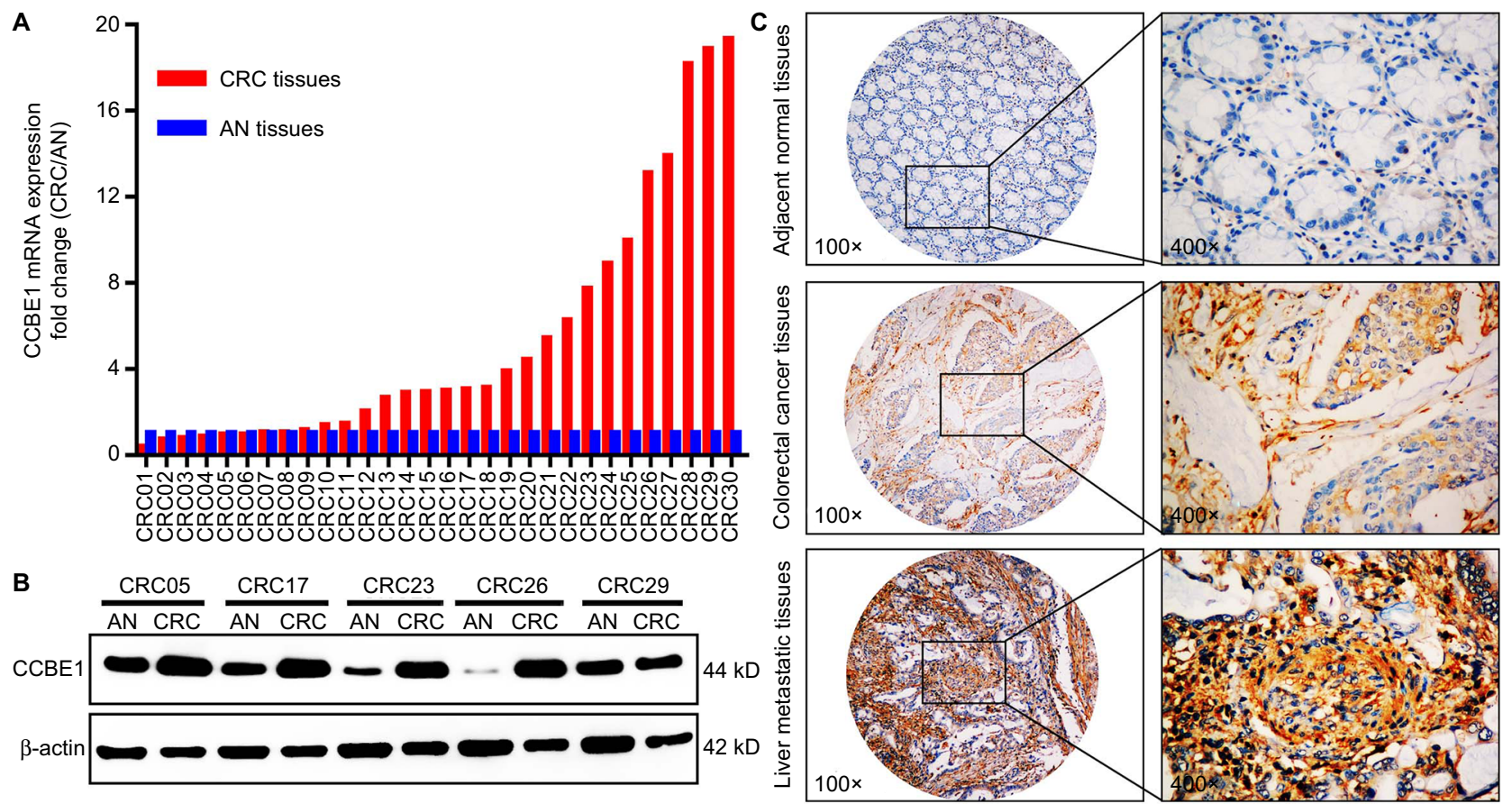

Figure I CCBEI expression in CRC patients.

Note: The expression of CCBEI was detected in 30 fresh CRC tissues and matched AN tissues by qRT-PCR (A), Western blotting (B) and IHC (C).

Abbreviations: AN, adjacent normal; CRC, colorectal cancer; IHC, immunohistochemistry; qRT-PCR, quantitative real-time PCR. 
CRC tissues is much stronger than that in paired primary CRC tissues and AN tissues (Figure 1C). These data indicate that CCBE1 is highly expressed in CRC tissues and may be associated with its invasion and metastasis.

\section{CCBEI expression correlates with clinicopathological features of CRC patients}

To analyze the association between CCBE1 and the clinicopathological features of $\mathrm{CRC}$, we examined the expression of CCBE1 in 204 CRC tissue samples by IHC. As shown in Figure 2, the ICH staining intensity of CCBE1 was scored as negative $(-)$, weak $(+)$, moderate $(++)$ and strong $(+++)$, and the percentage of positive cells was scored as $5 \%(-), 5$ $-30 \%(+), 31-50 \%(++)$ and $>50 \%(+++)$. The scores were calculated by multiplying these two values (ranging from 0 to 9). The protein expression in CRC specimens was divided into the low expression group $(<4)$ and the high expression group $(\geq 4)$ for further analysis. ${ }^{32,33}$ The protein expression in CRC specimens was divided into the low expression group (91, 44.6\%) and the high expression group (113, 55.4\%). Pearson's chi-squared test analysis showed that CCBE1 expression was significantly correlated with tumor differentiation $(P=0.003)$, TNM stage $(P=0.009)$, lymph node metastasis $(P<0.001)$, vascular invasion $(P=0.006)$, liver metastasis $(P=0.010)$ and serum carcinoembryonic antigen (CEA; $P=0.015$ ) expression, but was not associated with gender, age, primary tumor location, tumor size, serum CA19-9 expression, or postoperative chemotherapy (Table $1 ; P>0.05$ ).

\section{High CCBEI expression correlates with poor survival of $C R C$ patients}

The correlation between CCBE1 expression and patients' OS and DFS was estimated by Kaplan-Meier analyses. Kaplan-Meier analyses showed that CRC patients with higher expression of CCBE1 had poor OS and DFS compared with patients with lower expression $(P<0.001$ for both OS and DFS; Figure 3A); Moreover, tumor differentiation, lymph node metastasis, vascular invasion and liver metastasis were confirmed to be associated with patients' OS and DFS (Tables 2 and 3; Figure 3B-D; $P<0.01$ ). Univariate and multivariate analyses were performed to determine independent prognostic factors for CRC patients after surgery. Univariate analyses showed that CCBE1 expression $(P<0.001$ for OS and DFS $)$, tumor differentiation $(P<0.001$ for OS and DFS), lymph node metastasis $(P<0.001$ for OS and DFS), vascular invasion $(P<0.001$ for OS and DFS), liver metastasis $(P<0.001$ for OS and DFS $)$ and TNM stage ( $P=0.012$ for $\mathrm{OS}, P=0.005$ for DFS) were prognostic factors, and multivariate analyses showed that CCBE1 expression
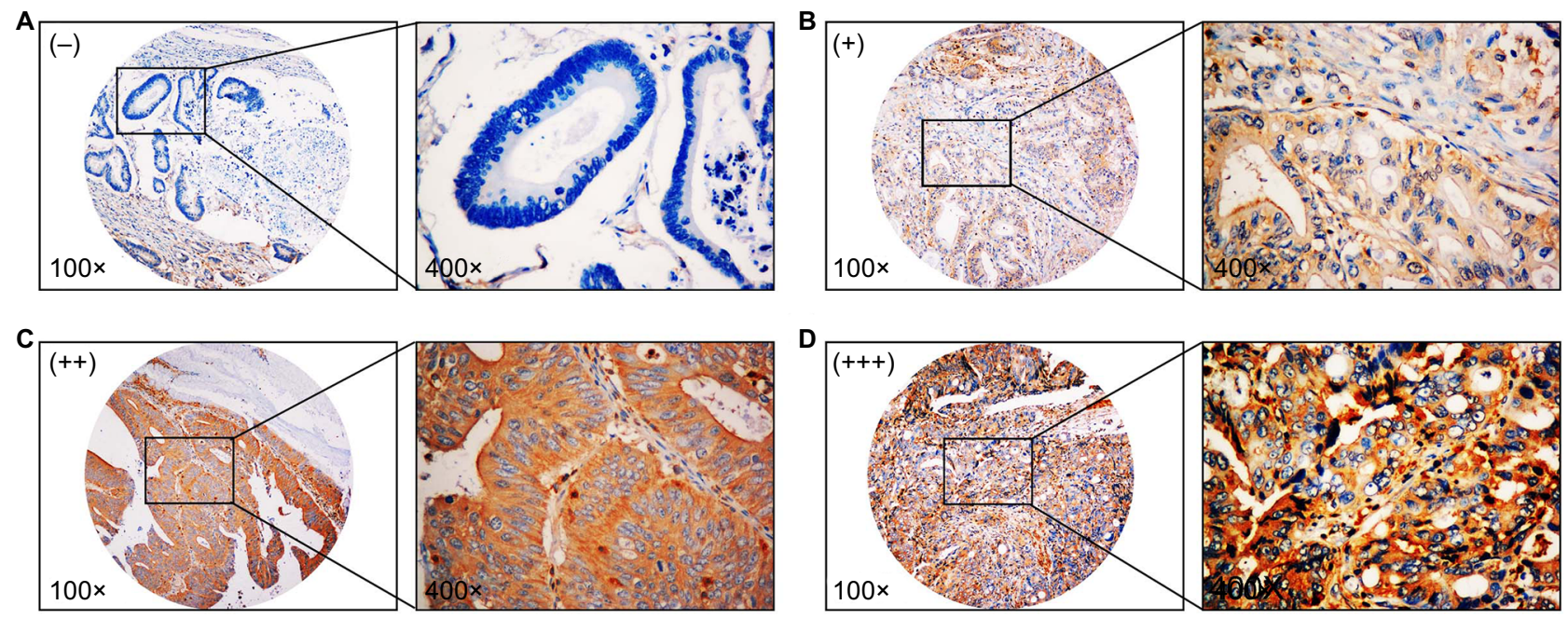

\begin{tabular}{|c|c|c|c|c|}
\hline \multirow{2}{*}{ CCBE1 staining } & \multicolumn{2}{|c|}{ Low expression } & \multicolumn{2}{|c|}{ High expression } \\
\hline & Negative (-) & Weak (+) & Moderate $(++)$ & Strong $(+++)$ \\
\hline CRC patients & $37(18.1 \%)$ & $54(26.5 \%)$ & $64(31.4 \%)$ & $49(24.0 \%)$ \\
\hline
\end{tabular}

Figure 2 CCBEI expression was detected in CRC patients by immunohistochemical staining.

Notes: (A) Thirty-seven cases of CCBEI negative staining of CRC tissues accounted for $18.1 \%$. (B) Fifty-four cases of CCBEI weak staining (+) of CRC tissues accounted for 26.5\%. (C) Sixty-four cases of CCBEI moderate staining of CRC tissues accounted for 31.4\%. (D) Forty-nine cases of CCBEI strong staining of CRC tissues accounted for $24.0 \%$. The protein expression in CRC specimens was divided into the low expression group (9I, 44.6\%) and the high expression group (II3, 55.4\%). Abbreviation: CRC, colorectal cancer. 
Table I Correlations of CCBEI expression with clinicopathological characteristics of CRC patients

\begin{tabular}{|c|c|c|c|c|}
\hline \multirow[t]{2}{*}{ Clinicopathological variables } & \multirow[t]{2}{*}{$\mathbf{n}$} & \multicolumn{2}{|l|}{ CCBEI } & \multirow[b]{2}{*}{$P$-value } \\
\hline & & Low expression & High expression & \\
\hline \multicolumn{5}{|l|}{ Gender } \\
\hline Male & 123 & 56 & 67 & \\
\hline Female & 81 & 35 & 46 & 0.744 \\
\hline \multicolumn{5}{|l|}{ Age (years) } \\
\hline$\leq 60$ & 84 & 41 & 43 & \\
\hline$>60$ & 120 & 50 & 70 & 0.312 \\
\hline \multicolumn{5}{|l|}{ Primary tumor location } \\
\hline $\mathrm{RC}$ & 98 & 44 & 54 & \\
\hline LC & 62 & 29 & 33 & \\
\hline Rectal & 44 & 18 & 26 & 0.833 \\
\hline \multicolumn{5}{|l|}{ Tumor differentiation } \\
\hline Well & 73 & 42 & 31 & \\
\hline Moderate & 84 & 37 & 47 & \\
\hline Poor & 47 & 12 & 35 & 0.003 \\
\hline \multicolumn{5}{|l|}{ Tumor size $(\mathrm{cm})$} \\
\hline$\leq 5$ & 81 & 39 & 42 & \\
\hline$>5$ & 123 & 52 & 71 & 0.409 \\
\hline \multicolumn{5}{|l|}{ TNM stage } \\
\hline I-II & 72 & 41 & 31 & \\
\hline III-IV & 132 & 50 & 82 & 0.009 \\
\hline \multicolumn{5}{|l|}{ Lymph node metastasis } \\
\hline Presence & 85 & 15 & 70 & \\
\hline Absence & 119 & 76 & 43 & $<0.001$ \\
\hline \multicolumn{5}{|l|}{ Vascular invasion } \\
\hline Presence & 63 & 19 & 44 & \\
\hline Absence & $|4|$ & 72 & 69 & 0.006 \\
\hline \multicolumn{5}{|l|}{ Liver metastasis } \\
\hline Presence & 59 & 18 & 41 & \\
\hline Absence & 145 & 73 & 72 & 0.010 \\
\hline \multicolumn{5}{|l|}{ Serum CEA } \\
\hline$\leq 5 \mathrm{ng} / \mathrm{mL}$ & 67 & 38 & 29 & \\
\hline$>5 \mathrm{ng} / \mathrm{mL}$ & 137 & 53 & 84 & 0.015 \\
\hline \multicolumn{5}{|l|}{ Serum CAI 9-9 } \\
\hline$\leq 37 \mathrm{U} / \mathrm{mL}$ & 55 & 30 & 25 & \\
\hline$>37 \mathrm{U} / \mathrm{mL}$ & 149 & 61 & 88 & 0.083 \\
\hline \multicolumn{5}{|l|}{ Postoperative chemotherapy } \\
\hline No & 35 & 17 & 18 & \\
\hline Yes & 169 & 74 & 95 & 0.604 \\
\hline
\end{tabular}

Notes: Low expression of CCBEI: IHC score $<4$; high expression of CCBEI: IHC score $\geq 4$. Significant results $(P<0.05)$ are given in bold.

Abbreviations: CEA, carcinoembryonic antigen; CRC, colorectal cancer; IHC, immunohistochemistry; LC, left-sided colon; RC, right-sided colon.

$(P<0.001$ for OS, $P=0.028$ for DFS $)$, tumor differentiation $(P=0.001$ for OS, $P<0.001$ for DFS), lymph node metastasis (not significant $[\mathrm{NS}]$ for OS, $P=0.044$ for DFS), vascular invasion $(P<0.001$ for OS, NS for DFS) were independent prognostic factors in CRC patients after surgery (Tables 2 and 3). In The Cancer Genome Atlas (TCGA) CRC data, the patients were divided into the low expression group and the high expression group according to the same median value. In TCGA database analysis, patients with high CCBE1 levels had a worse prognosis than patients with low CCBE1 levels
(OS: log-rank $P=6 \mathrm{e}-04$, HR $[$ high $]=2.4, P[\mathrm{HR}]=0.00084$; DFS: log-rank $P=0.0081$, HR [high $]=1.9, P[\mathrm{HR}]=0.0093$; Figure 4). These results fully demonstrated that CCBE1 expression was closely correlated with poor survival and could be used as a novel independent prognostic biomarker for CRC patients after surgery.

\section{Discussion}

$\mathrm{CRC}$ is the third most common malignant cancer and one of the main causes of cancer-related death worldwide. ${ }^{1}$ 

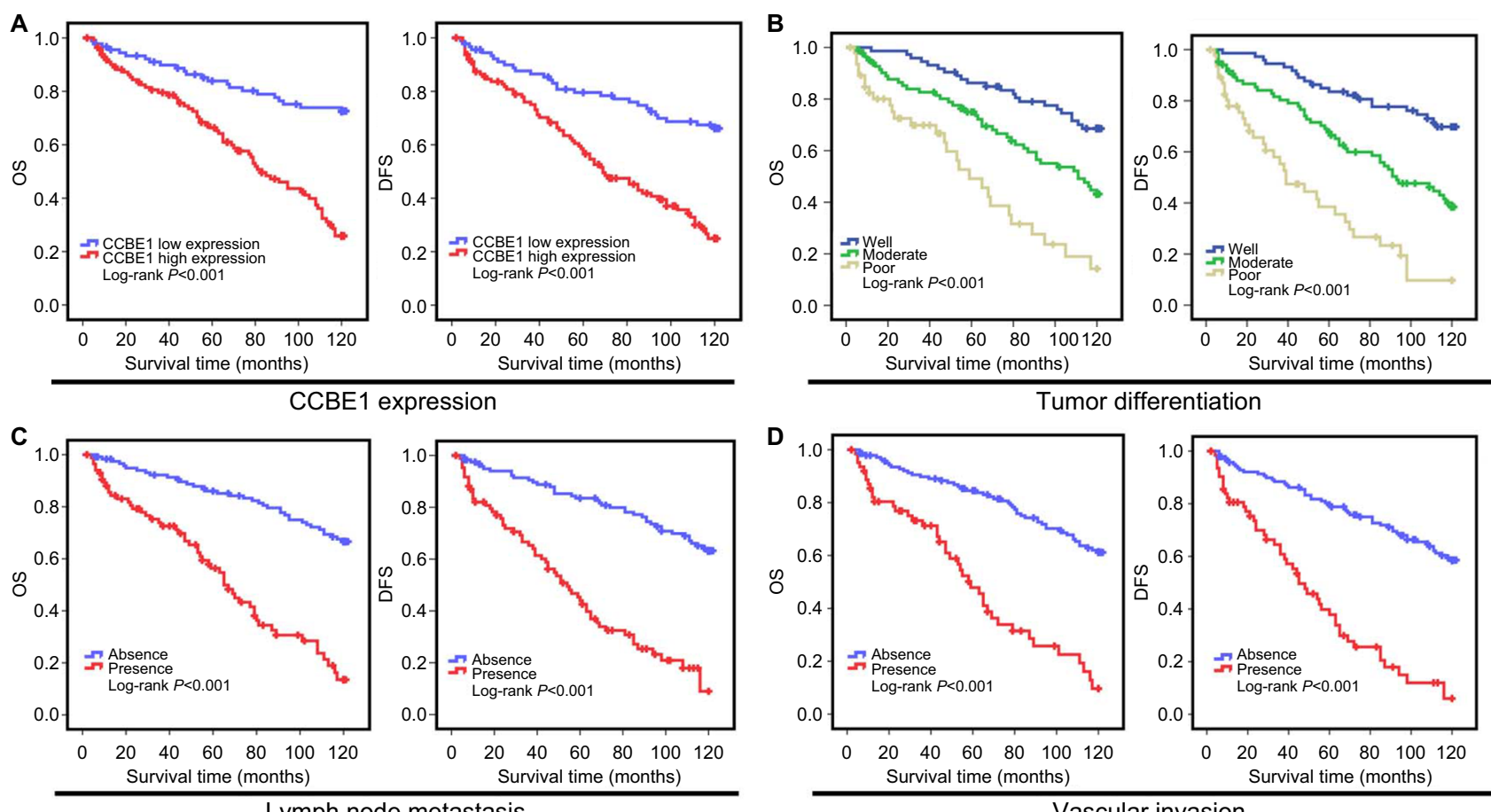

Lymph node metastasis

Vascular invasion

Figure 3 Kaplan-Meier OS and DFS analyses of CRC.

Note: Results indicated that CCBEI expression (A), tumor differentiation (B), lymph node metastasis (C) and vascular invasion (D) were associated with CRC patients' prognosis (all $P<0.001$ ).

Abbreviations: CRC, colorectal cancer; DFS, disease-free survival; OS, overall survival.

The bad prognosis of patients with CRC is largely due to the metastatic progression. ${ }^{2}$ In general, the metastatic process involves the spread of tumor cells, vascular invasion, lymph node metastasis and growth of new cancer cell colonies. ${ }^{2,4,5,7,8,34}$ Therefore, it is necessary to identify effective predictors to help patients choose appropriate treatments and monitoring. As a newly discovered important lymphangiogenesis and pro-angiogenic factor, $\mathrm{CCBE} 1$ has received increasing attention in cancer research. ${ }^{21,23-27}$ In this study, data analyzed from 30 fresh CRC tissues and paired AN tissues indicated that CCBE1 is highly expressed in CRC tissues and may be associated with tumor malignancy, invasion and metastasis. This result is consistent with the findings of Zhang and $\mathrm{Liu}^{27}$ and Tian et $\mathrm{al},{ }^{25}$ which showed that the upregulation of CCBE1 aggravates lymphatic metastasis of rectal cancer and enhances tumor angiogenesis of GISTs. However, CCBE1 is similar to other oncogenes, has tissue specificity and has different expression levels and effects in different tumors. For instance, Barton et $\mathrm{al}^{21}$ supported CCBE1 as a potential tumor suppressor in ovarian cancer, and Mesci et $\mathrm{al}^{23}$ showed that CCBE1 exerts a tumor suppressive effect in breast cancer by in vitro invasion assay of human breast cancer cell lines. In addition, lymphatic metastasis and vascular invasion are the main means of CRC spread and can significantly affect the prognosis of patients..$^{35}$ Previous studies found that CCBE1 is a secreted molecule involved in lymphangiogenesis, ${ }^{14,36}$ and congenital CCBE1 mutations have been shown to cause Hennekam syndrome. ${ }^{36,37}$ To confirm the relationship between CCBE1 expression and prognosis and clinicopathological features of CRC, 204 cases of CRC tissues were stained to analyze. IHC results showed that high expression of CCBE1 was observed in 55.4\% (113/204) CRC patients. Pearson's chi-squared test analysis showed that CCBE1 expression was significantly correlated with tumor differentiation, TNM stage, lymph node metastasis, vascular invasion, liver metastasis and serum CEA, but was not associated with gender, age, primary tumor location, tumor size, serum CA19-9 level or postoperative chemotherapy. The abovementioned results indicated that the expression level of CCBE1 was closely related to CRC invasion and metastasis. It is well known that tumor differentiation, lymph node metastasis, vascular invasion, organ metastasis and TNM staging are key factors influencing the progression and survival prognosis of CRC patients. ${ }^{2,30,34,38}$ In the present study, our data also indicated that tumor differentiation, lymph node metastasis, 
Table 2 Univariable and multivariable analysis of OS and clinicopathological variables of CRC patients

\begin{tabular}{|c|c|c|c|c|c|}
\hline \multirow[t]{2}{*}{ Clinicopathological variables } & \multirow[t]{2}{*}{$\mathbf{n}$} & \multicolumn{2}{|l|}{ Univariable analysis } & \multicolumn{2}{|c|}{ Multivariable analysis } \\
\hline & & HR (95\% Cl) & P-Value & HR (95\% Cl) & $P$-value \\
\hline \multicolumn{6}{|l|}{ Gender } \\
\hline Male & 123 & I & & & \\
\hline Female & 81 & I.I 48 (0.749-I.758) & 0.527 & & NA \\
\hline \multicolumn{6}{|l|}{ Age (years) } \\
\hline$\leq 60$ & 84 & I & & & \\
\hline$>60$ & 120 & $1.300(0.853-1.98 I)$ & 0.222 & & NA \\
\hline \multicolumn{6}{|l|}{ Primary tumor location } \\
\hline $\mathrm{RC}$ & 98 & I & & & \\
\hline LC & 62 & $1.165(0.904-1.502)$ & 0.238 & & NA \\
\hline Rectal & 44 & & & & \\
\hline \multicolumn{6}{|l|}{ Tumor differentiation } \\
\hline Well & 73 & 1 & & 1 & \\
\hline Moderate & 84 & $2.287(1.716-3.048)$ & $<0.001$ & $1.656(1.232-2.225)$ & 0.001 \\
\hline Poor & 47 & & & & \\
\hline \multicolumn{6}{|l|}{ Tumor size $(\mathrm{cm})$} \\
\hline$\leq 5$ & 81 & 1 & & & \\
\hline$>5$ & 123 & $1.318(0.858-2.024)$ & 0.208 & & NA \\
\hline \multicolumn{6}{|l|}{ TNM stage } \\
\hline I-II & 72 & 1 & & & \\
\hline III-IV & 132 & $1.766(1.130-2.758)$ & 0.012 & & NS \\
\hline \multicolumn{6}{|l|}{ Lymph node metastasis } \\
\hline Presence & 85 & I & & & \\
\hline Absence & 119 & $0.222(0.143-0.342)$ & $<0.001$ & & NS \\
\hline \multicolumn{6}{|l|}{ Vascular invasion } \\
\hline Presence & 63 & I & & I & \\
\hline Absence & 141 & $0.235(0.153-0.360)$ & $<0.001$ & $0.397(0.25 \mathrm{I}-0.629)$ & $<0.001$ \\
\hline \multicolumn{6}{|l|}{ Liver metastasis } \\
\hline Presence & 59 & I & & & \\
\hline Absence & 145 & $0.242(0.158-0.372)$ & $<0.001$ & & NS \\
\hline \multicolumn{6}{|l|}{ Serum CEA } \\
\hline$\leq 5 \mathrm{ng} / \mathrm{mL}$ & 67 & 1 & & & \\
\hline$>5 \mathrm{ng} / \mathrm{mL}$ & 137 & $1.267(0.812-1.976)$ & 0.297 & & NA \\
\hline \multicolumn{6}{|l|}{ Serum CAI9-9 } \\
\hline$\leq 37 \mathrm{U} / \mathrm{mL}$ & 55 & 1 & & & \\
\hline$>37 \mathrm{U} / \mathrm{mL}$ & 149 & $0.931(0.591-1.466)$ & 0.757 & & NA \\
\hline \multicolumn{6}{|l|}{ Postoperative chemotherapy } \\
\hline No & 35 & I & & & \\
\hline Yes & 169 & $1.035(0.611-1.753)$ & 0.897 & & NA \\
\hline \multicolumn{6}{|l|}{ CCBEI expression } \\
\hline Low & 91 & I & & I & \\
\hline High & 113 & $3.660(2.27 I-5.900)$ & $<0.001$ & $2.614(1.594-4.288)$ & $<0.001$ \\
\hline
\end{tabular}

Notes: Bold values indicate statistical significance $P<0.05$.

Abbreviations: CEA, carcinoembryonic antigen; CRC, colorectal cancer; LC, left-sided colon; NA, not applicable; NS, not significant; OS, overall survival; RC, right-sided colon.

vascular invasion and liver metastasis were associated with patients' poor OS and DFS. Most importantly, we found that high expression of CCBE1 was significantly associated with CRC patients' poor OS and DFS, and OS analysis of 267 CRC patients from the TCGA database showed the same trend. Previous studies showed that the upregulation of CCBE1 was correlated with poorer survival of rectal cancer ${ }^{26}$ and GISTs, ${ }^{25}$ similar to our results. However, the tumor-promoting or tumor suppressor function of CCBE1 has background specificity, even in cancers of the same tissue origin. Mesci et $\mathrm{al}^{23}$ provided the first report on the loss of CCBE1 in breast cancer, and Barton et a ${ }^{21}$ described the loss of CCBE1 in ovarian cancers. Their results showed a correlation with poorer survival with lower CCBE1 
Table 3 Univariable and multivariable analysis of DFS and clinicopathological variables of CRC patients

\begin{tabular}{|c|c|c|c|c|c|}
\hline \multirow[t]{2}{*}{ Clinicopathological variables } & \multirow[t]{2}{*}{$\mathbf{n}$} & \multicolumn{2}{|l|}{ Univariable analysis } & \multicolumn{2}{|c|}{ Multivariable analysis } \\
\hline & & HR $(95 \% \mathrm{Cl})$ & $P$-value & HR (95\% Cl) & $P$-value \\
\hline \multicolumn{6}{|l|}{ Gender } \\
\hline Male & 123 & I & & & \\
\hline Female & 81 & $1.257(0.832-1.899)$ & 0.278 & & NA \\
\hline \multicolumn{6}{|l|}{ Age (years) } \\
\hline$\leq 60$ & 84 & 1 & & & \\
\hline$>60$ & 120 & $1.337(0.894-2.001)$ & 0.158 & & NA \\
\hline \multicolumn{6}{|l|}{ Primary tumor location } \\
\hline RC & 98 & 1 & & & \\
\hline LC & 62 & $1.194(0.938-1.519)$ & 0.150 & & NA \\
\hline Rectal & 44 & & & & \\
\hline \multicolumn{6}{|l|}{ Tumor differentiation } \\
\hline Well & 73 & I & & I & \\
\hline Moderate & 84 & $2.538(1.923-3.350)$ & $<0.001$ & $1.868(1.40 \mathrm{I}-2.492)$ & $<0.001$ \\
\hline Poor & 47 & & & & \\
\hline \multicolumn{6}{|l|}{ Tumor size (cm) } \\
\hline$\leq 5$ & 81 & 1 & & & \\
\hline$>5$ & 123 & $1.099(0.736-1.64 I)$ & 0.643 & & NA \\
\hline \multicolumn{6}{|l|}{ TNM stage } \\
\hline I-II & 72 & I & & & \\
\hline III-IV & 132 & $1.847(1.203-2.837)$ & 0.005 & & NS \\
\hline \multicolumn{6}{|l|}{ Lymph node metastasis } \\
\hline Presence & 85 & I & & I & \\
\hline Absence & 119 & $0.216(0.142-0.331)$ & $<0.001$ & $0.505(0.260-0.982)$ & 0.044 \\
\hline \multicolumn{6}{|l|}{ Vascular invasion } \\
\hline Presence & 63 & I & & & \\
\hline Absence & 141 & $0.220(0.145-0.333)$ & $<0.001$ & & NS \\
\hline \multicolumn{6}{|l|}{ Liver metastasis } \\
\hline Presence & 59 & I & & & \\
\hline Absence & 145 & $0.225(0.149-0.34 I)$ & $<0.001$ & & NS \\
\hline \multicolumn{6}{|l|}{ Serum CEA } \\
\hline$\leq 5 \mathrm{ng} / \mathrm{mL}$ & 67 & 1 & & & \\
\hline$>5 \mathrm{ng} / \mathrm{mL}$ & 137 & $1.264(0.825-1.937)$ & 0.282 & & NA \\
\hline \multicolumn{6}{|l|}{ Serum CAI9-9 } \\
\hline$\leq 37 \mathrm{U} / \mathrm{mL}$ & 55 & 1 & & & \\
\hline$>37 \mathrm{U} / \mathrm{mL}$ & 149 & $0.886(0.575-1.363)$ & 0.581 & & NA \\
\hline \multicolumn{6}{|l|}{ Postoperative chemotherapy } \\
\hline No & 35 & I & & & \\
\hline Yes & 169 & $0.893(0.552-1.444)$ & 0.644 & & NA \\
\hline \multicolumn{6}{|l|}{ CCBEI expression } \\
\hline Low & 91 & I & & 1 & \\
\hline High & 113 & $3.009(1.942-4.661)$ & $<0.001$ & 1.740 (1.060-2.857) & 0.028 \\
\hline
\end{tabular}

Notes: Bold values indicate statistical significance $P<0.05$.

Abbreviations: CEA, carcinoembryonic antigen; CRC, colorectal cancer; DFS, disease-free survival; LC, left-sided colon; NA, not applicable; NS, not significant; RC, rightsided colon.

expression, which is contrary to our results. Univariate and multivariate analyses showed that CCBE1 expression, tumor differentiation, lymph node metastasis and vascular invasion were independent prognostic factors in CRC patients after surgery. These results fully demonstrated that the presence of CCBE1 was closely correlated with poor survival and could be used as a novel independent prognostic biomarker for CRC patients after surgery. However, our study only initially assessed the clinical value of CCBE1 in CRC patients. To confirm the prognostic value of CCBE1 in CRC patients, further multicenter studies are needed to validate our observations. 

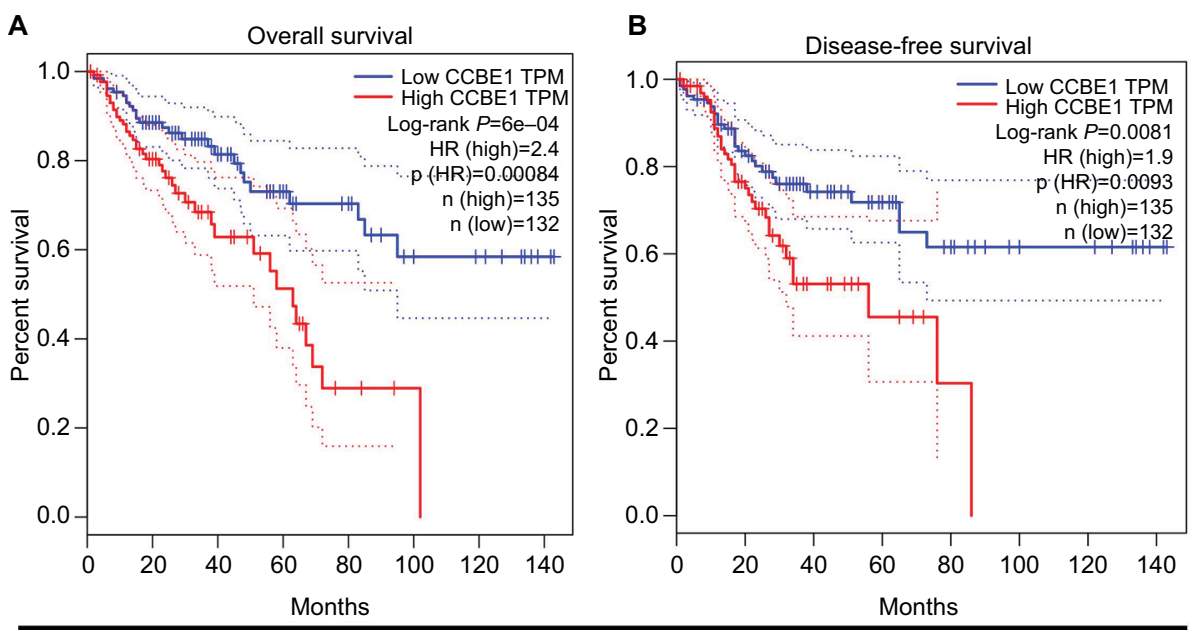

TCGA CRC data

Figure 4 Kaplan-Meier OS (A) and DFS (B) curves of the TCGA CRC cohort show that patients with high CCBEI level had a worse prognosis than patients with low CCBEI level (OS: log-rank $P=6 \mathrm{e}-04, \mathrm{HR}$ [high] $=2.4, P[\mathrm{HR}]=0.00084$; DFS: log-rank $P=0.008 \mathrm{I}$, HR [high] $=1.9, P[\mathrm{HR}]=0.0093$ ).

Abbreviations: CRC, colorectal cancer; DFS, disease-free survival; OS, overall survival; TCGA, The Cancer Genome Atlas; TPM, transcripts per million.

\section{Conclusion}

Our study suggests that CCBE1 is a highly expressed oncogene in CRC patients. High expression of CCBE1 is significantly related to tumor differentiation, TNM stage, lymph node metastasis, vascular invasion, liver metastasis, serum CEA and poor prognosis. In addition, our findings indicated that CCBE1 was closely correlated with poor OS and DFS and could be used as a novel independent prognostic biomarker for CRC patients after surgery. Of course, further investigations are needed to validate our findings.

\section{Acknowledgment}

The authors would like to thank TCGA and all the patients and their families who agreed to participate in this study.

\section{Author contributions}

CGY conceived and designed the study. YRZ and HL performed the experiments and YRZ wrote the manuscript. LMX and CGY assisted with experimental performance. YRZ, CGJ and ZPZ assisted with the data analysis. YRZ and CGY modified the manuscript. All the authors contributed toward data analysis, drafting and revising the paper, gave final approval of the version to be published and agreed to be accountable for all aspects of the work.

\section{Disclosure}

The authors report no conflicts of interest in this work.

\section{References}

1. Torre LA, Bray F, Siegel RL, Ferlay J, Lortet-Tieulent J, Jemal A. Global cancer statistics, 2012. CA Cancer J Clin. 2015;65(2):87-108.
2. Sun ZQ, Ma S, Zhou QB, et al. Prognostic value of lymph node metastasis in patients with $\mathrm{T} 1$-stage colorectal cancer from multiple centers in China. World J Gastroenterol. 2017;23(48):8582-8590.

3. Mcallister SS, Weinberg RA. The tumour-induced systemic environment as a critical regulator of cancer progression and metastasis. Nat Cell Biol. 2014;16(8):717-727.

4. Valastyan S, Weinberg RA. Tumor metastasis: molecular insights and evolving paradigms. Cell. 2011;147(2):275-292.

5. Hanahan D, Weinberg RA. Hallmarks of cancer: the next generation. Cell. 2011;144(5):646-674.

6. Liu H, Liu Z, Li K, et al. TBL1XR1 predicts isolated tumor cells and micrometastasis in patients with TNM stage I/II colorectal cancer. $J$ Gastroenterol Hepatol. 2017;32(9):1570-1580.

7. Shibue T, Weinberg RA. Metastatic colonization: settlement, adaptation and propagation of tumor cells in a foreign tissue environment. Semin Cancer Biol. 2011;21(2):99-106.

8. Chen W, Zheng R, Baade PD, et al. Cancer statistics in China, 2015. CA Cancer J Clin. 2016;66(2):115-132.

9. Chen W. Cancer statistics: updated cancer burden in China. Chin J Cancer Res. 2015;27(1):1.

10. You WC, Jin F, Devesa S, et al. Rapid increase in colorectal cancer rates in urban Shanghai, 1972-97, in relation to dietary changes. $J$ Cancer Epidemiol Prev. 2002;7(3):143-146.

11. Yamamoto F, Yamamoto M. Scanning copy number and gene expression on the 18q21-qter chromosomal region by the systematic multiplex PCR and reverse transcription-PCR methods. Electrophoresis. 2007;28(12):1882-1895.

12. Furtado J, Bento M, Correia E, Inácio JM, Belo JA. Expression and function of Ccbe1 in the chick early cardiogenic regions are required for correct heart development. PLoS One. 2014;9(12):e115481.

13. Zou Z, Enis DR, Bui H, et al. The secreted lymphangiogenic factor CCBE1 is essential for fetal liver erythropoiesis. Blood. 2013;121(16):3228-3236.

14. Hogan BM, Bos FL, Bussmann J, et al. Ccbe1 is required for embryonic lymphangiogenesis and venous sprouting. Nat Genet. 2009;41(4):396-398.

15. Bos FL, Caunt M, Peterson-Maduro J, et al. CCBE1 is essential for mammalian lymphatic vascular development and enhances the lymphangiogenic effect of vascular endothelial growth factor-C in vivo. Circ Res. 2011;109(5):486-491.

16. Hägerling R, Pollmann C, Andreas M, et al. A novel multistep mechanism for initial lymphangiogenesis in mouse embryos based on ultramicroscopy. EMBO J. 2013;32(5):629-644. 
17. Weijts BG, van Impel A, Schulte-Merker S, de Bruin A. Atypical E2fs control lymphangiogenesis through transcriptional regulation of $\mathrm{Ccbe} 1$ and Flt4. PLoS One. 2013;8(9):e73693.

18. Astin JW, Haggerty MJ, Okuda KS, et al. Vegfd can compensate for loss of Vegfc in zebrafish facial lymphatic sprouting. Development. 2014;141(13):2680-2690.

19. Jeltsch M, Jha SK, Tvorogov D, et al. CCBE1 enhances lymphangiogenesis via $\mathrm{A}$ disintegrin and metalloprotease with thrombospondin motifs-3-mediated vascular endothelial growth factor-C activation. Circulation. 2014;129(19):1962-1971.

20. Pollmann C, Hägerling R, Kiefer F. Visualization of lymphatic vessel development, growth, and function. Adv Anat Embryol Cell Biol. 2014;214:167-186.

21. Barton CA, Gloss BS, Qu W, et al. Collagen and calcium-binding EGF domains 1 is frequently inactivated in ovarian cancer by aberrant promoter hypermethylation and modulates cell migration and survival. Br J Cancer. 2010;102(1):87-96.

22. Li P, Cong Z, Qiang Y, et al. Clinical significance of CCBE1 expression in lung cancer. Mol Med Rep. 2018;17(2):2107-2112.

23. Mesci A, Huang X, Taeb S, et al. Targeting of CCBE1 by miR330-3p in human breast cancer promotes metastasis. Br J Cancer. 2017;116(10):1350-1357.

24. MesciA, Liu SK. Collagen and Calcium Binding EGF Domains 1 (CCBE1) in cancer - a new role past lymphatics? Oncoscience. 2017;4(11-12):168-169.

25. Tian GA, Zhu CC, Zhang XX, et al. CCBE1 promotes GIST development through enhancing angiogenesis and mediating resistance to imatinib. Sci Rep. 2016;6:31071.

26. Guo RL, Wang XR, Wang QG, et al. The regulatory role of SLP-2 and mechanism on CCBE1 gene expression in rectal carcinoma and adjacent lymphatic tube tissues. Eur Rev Med Pharmacol Sci. 2018;22(1):87-94.

27. Zhang L, Liu FJ. Expression of SLP-2 gene and CCBE1 are associated with prognosis of rectal cancer. Eur Rev Med Pharmacol Sci. 2017;21(6):1214-1218
28. Pei Q, Zhu H, Tan F, et al. Intravascular emboli is an independent risk factor for the prognosis of stage III colorectal cancer patients after radical surgery. Oncotarget. 2016;7(35):57268-57276.

29. Gu D, Szallasi A. Thrombocytosis Portends Adverse Prognosis in Colorectal Cancer: A Meta-Analysis of 5,619 Patients in 16 Individual Studies. Anticancer Res. 2017;37(9):4717-4726.

30. Croner RS, Förtsch T, Brückl WM, et al. Molecular signature for lymphatic metastasis in colorectal carcinomas. Ann Surg. 2008;247(5):803-810.

31. Sun Z, Ou C, Ren W, Xie X, Li X, Li G. Downregulation of long non-coding RNA ANRIL suppresses lymphangiogenesis and lymphatic metastasis in colorectal cancer. Oncotarget. 2016;7(30): 47536-47555

32. Terpe HJ, Störkel S, Zimmer U, et al. Expression of CD44 isoforms in renal cell tumors. Positive correlation to tumor differentiation. Am J Pathol. 1996;148(2):453-463.

33. Tao YM, Huang JL, Zeng S, et al. BTB/POZ domain-containing protein 7: epithelial-mesenchymal transition promoter and prognostic biomarker of hepatocellular carcinoma. Hepatology. 2013;57(6):2326-2337.

34. Naxerova K, Reiter JG, Brachtel E, et al. Origins of lymphatic and distant metastases in human colorectal cancer. Science. 2017;357(6346): 55-60.

35. Tacconi C, Correale C, Gandelli A, et al. Vascular endothelial growth factor $\mathrm{C}$ disrupts the endothelial lymphatic barrier to promote colorectal cancer invasion. Gastroenterology. 2015;1481451(7):1438-1451.

36. Alders M, Hogan BM, Gjini E, et al. Mutations in CCBE1 cause generalized lymph vessel dysplasia in humans. Nat Genet. 2009;41(12): $1272-1274$

37. Jackson CC, Best L, Lorenzo L, et al. A Multiplex Kindred with Hennekam Syndrome due to Homozygosity for a CCBE1 Mutation that does not Prevent Protein Expression. J Clin Immunol. 2016;36(1): 19-27.

38. Arteaga A, Leach SD, Harari PM. Spreading Colon Cancer Can Bypass Lymph Nodes. Cancer Discov. 2017;7(9):924-925.
Cancer Management and Research

\section{Publish your work in this journal}

Cancer Management and Research is an international, peer-reviewed open access journal focusing on cancer research and the optimal use of preventative and integrated treatment interventions to achieve improved outcomes, enhanced survival and quality of life for the cancer patient. The manuscript management system is completely online and includes

\section{Dovepress}

a very quick and fair peer-review system, which is all easy to use. Visit http://www.dovepress.com/testimonials.php to read real quotes from published authors. 